\title{
Consensus statement regarding minimally invasive surgery during the COVID-19 pandemic
}

\author{
Compiled on behalf of SASES EXCO by D Nel, ${ }^{1,4}$ R Oodit, ${ }^{2,4}$ H Bougard,,4 E Panieri ${ }^{3,4}$ \\ Surgical Registrar, ${ }^{1}$ Consultant Surgeons in Private ${ }^{2}$ and State ${ }^{3}$ sectors affiliated to University of Cape Town ${ }^{4}$
}

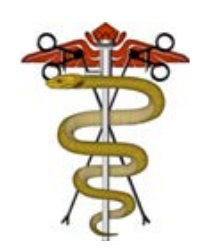

\section{Introduction}

SASES is committed to providing guidelines and support to our members to ensure that patients receive the best available care. The COVID-19 pandemic has placed health systems worldwide under unprecedented strain.

COVID-19-infected patients will present with conditions requiring surgical intervention. The purpose of this document is to address concerns relating specifically to laparoscopic surgery in COVID-19-infected patients.

SASES has made the following surgical recommendations based on current available evidence. We will endeavour to update this document as new evidence develops.

\section{Is laparoscopic surgery appropriate for the patient who is COVID-19 positive?}

\section{Elective presentation}

COVID-19-infected patients should not undergo elective surgery, regardless if they are symptomatic or not, until they have completely recovered.

\section{Emergency presentation}

\section{Non-operative management}

Wherever possible, a non-operative management strategy should be considered. ${ }^{1}$ Clinical discretion is paramount and the final therapeutic decision rests on the treating surgeon. Appropriate surgical intervention should not be deferred on the basis of SARS-CoV-2 positivity alone.

\section{Operative management}

Should a COVID-19-infected patient require an intraabdominal procedure, there is no reason to believe that laparoscopy is any more or less harmful than a laparotomy to the patient on the basis of COVID-19 disease alone.

Laparoscopy has the theoretical advantage of fewer postoperative respiratory complications, faster recovery and early discharge from hospital. ${ }^{2}$ These have to be considered in the clinical context of each case, and weighed up against the anticipated duration of surgery.

The surgeon should be competent in performing the procedure safely and in the shortest time possible; an open approach should be performed if laparoscopy will add significant time to the procedure or has doubtful advantages in terms of morbidity and length of stay.

\section{Is laparoscopic surgery safe for the surgeon and the theatre staff?}

Although there is an obvious theoretical risk to theatre staff during laparoscopy, no case of COVID-19 transmission to theatre staff during laparoscopy has been documented to date. $^{3}$ In the vast majority of cases of infected healthcare workers, it is impossible to isolate a single likely exposure point.

Energy devices, including electrocautery and ultrasonic dissectors, have been shown to cause aerosolisation of HPV, HBV and HIV particles during open and MIS. ${ }^{4}$ It is reasonable and prudent to assume that aerosolisation of COVID-19 particles would occur in a similar way.

COVID-19 RNA has been isolated from the respiratory tract, the entire gastrointestinal tract, bile, faeces, and, critically, also in peritoneal fluid. ${ }^{5,6}$ What is not known is whether these RNA fragments are virulent.

The risk of staff exposure to COVID-19 may be higher in laparoscopy due to the concentration of suspended viral particles in the pneumoperitoneum, with potential risk during release of $\mathrm{CO}^{2}$ when smoke venting, and with specimen or trocar removal. ${ }^{7}$

Uncontrolled dispersion of aerosolised virus within the operating room potentially places other members of the team at risk of exposure even when not directly involved in the surgical procedure.

Open surgery however does not preclude the risk of virus aerosolisation, especially where electrocautery is used, and indeed smoke plumes may be less easily controlled than those within a closed body cavity, as in laparoscopy. Additionally, it is more likely to expose surgical staff to direct blood or fluid contact. ${ }^{8}$

\section{Recommendations for the performance of laparoscopic surgery in a COVID-19 positive or person under investigation (PUI) patient}

Minimise the number of staff in the theatre room at all times. Do not enter the OR during intubation and leave prior to extubation. 
PPE recommendations for operating staff

- Waterproof gowns

- $\quad$ N95 or better respirator masks

- Face shields or goggles to be worn at all times

- Apron

- Double gloving

- Adherence of outer glove to gown to achieve single doffing

- Waterproof overshoes or washable footwear

- Donning and doffing of PPE to follow institutional guidelines $^{9}$

\section{Performance of the laparoscopic procedure}

- Limit accidental gas leak at port sites.

- Make skin and sheath incisions of appropriate size to ensure snug trocar fit.

- Consider a balloon port at the Hasson access site.

- Suture skin around leaking port sites, or pack with a gauze swab soaked in liquid paraffin.

- Ensure port sites remain clean and dry to avoid dispersion of fluids if inadvertent gas leak around trocar.

- Minimum pressure to maintain pneumoperitoneum $(10-12 \mathrm{mmHg})$

- Hand ports significantly increase gas leaks, and should be avoided.

- Cautery set to lowest effective setting and used as little as possible.

- Smoke extraction from port with High-Efficiency Particulate Air (HEPA) filter only. ${ }^{10}$

- Standard wall suction units should not be used to desufflate the peritoneal cavity.

- Minimise instrument exchange through ports.

- Deflate peritoneal cavity fully before specimen removal/ extracorporeal anastomosis; reinflate afterwards if necessary.

- Careful and complete deflation before trocar removal at the end of the case, or before conversion to open.

- The patient should be flat and the least dependent port should be utilised for desufflation.

- Avoid leaving drains.

\section{Gas filters and desufflation}

During desufflation, all escaping $\mathrm{CO}^{2}$ gas and smoke should be captured with an ultra-filtration system.

Some laparoscopy units have a desufflation mode, which can be used in conjunction with a (HEPA) filter to prevent contamination of the insufflator.

If the insufflator being used does not have a desufflation feature, close the valve on the working port that is being used for insufflation, before the flow of $\mathrm{CO}^{2}$ on the insufflator is turned off (even if there is an in-line filter in the tubing). Without taking this precaution contaminated intraabdominal $\mathrm{CO}^{2}$ can be pushed into the insufflator when the intra-abdominal pressure is higher than the pressure within the insufflator.

\section{Gas evacuation systems}

We are unable to recommend a specific gas evacuation system, as superior to another. There are various methods to remove $\mathrm{CO}^{2}$ and smoke safely during laparoscopic procedures. ${ }^{10,11}$ A number of commercial evacuation systems have been designed for active smoke extraction.

\section{Conclusion}

Laparoscopy is not contraindicated in procedures where it results in an unequivocal clinical benefit over an open approach, provided the surgeon and health facility are able to comply with the precautionary measures outlined above.

Surgical teams are urged to employ all available recommended safety measures because of the potential increased risks of viral dissemination and exposure during surgery and in particular minimal access surgery where pressurised gas insufflation is used.

\section{Key links}

- SAGES/EAES guideline for MIS during COVID-19: https://www.sages.org/recommendations-surgicalresponse-covid-19/

- SAGES resources for smoke and gas evacuation: https:// www.sages.org/resources-smoke-gas-evacuationduring-open-laparoscopic-endoscopic-procedures/

- Method for smoke evacuation in laparoscopic surgery for suspected COVID-19 patients: https://eaes.eu/ a-low-cost-safe-and-effective-method-for-smokeevacuation-in-laparoscopic-surgery-for-suspectedcoronavirus-patients/a-low-cost-safe-and-effective-method-for-smoke-evacuation-in-laparoscopicsurgery-for-suspected-coronavirus- patients/

\section{REFERENCES}

1. Updated Intercollegiate General Surgery Guidance on COVID-19. [Internet]. Royal College of Surgeons of England. 2020. Available from: https://www.rcseng.ac.uk/coronavirus/ joint-guidance-for- surgeons-v2/. (Accessed 9 May 2020).

2. Vigneswaran Y, Prachand VN, Posner MC, et al. What is the appropriate use of laparoscopy over open procedures in the current COVID-19 climate? J Gastrointest Surg. 2020 Apr 13:16. https://doi.org/10.1007/s11605-020-04592-9. [Epub ahead of print].

3. Francis N, Dort J, Cho E, et al. SAGES and EAES recommendations for minimally invasive surgery during COVID-19 pandemic. Surgical Endoscopy. 2020 Apr 22:1-5.

4. Veziant J, Bourdel N, Slim K. Risks of viral contamination in healthcare professionals during laparoscopy in the Covid-19 pandemic. Journal of Visceral Surgery. 2020 Apr 17. https://doi. org/10.1016/j.jviscsurg.2020.04.010. [Epub ahead of print].

5. Siew C Ng, Tilg H. COVID-19 and the gastrointestinal tract: more than meets the eye. Gut. 2020 Jun;69(6):973-974. https:// doi.org/10.1136/gutjnl-2020-321195. [Epub 2020 Apr 9].

6. Coccolini F, Tartaglia D, Puglisi A, et al (in press). SARSCoV-2 is present in peritoneal fluid in COVID-19 patients. Annals of Surgery. Available from: https://journals.lww. com/annalsofsurgery/Documents/SARS-CoV- $2 \% 20$ is $\% 20$ present $\% 20 \mathrm{in} \% 20$ peritoneal $\% 20$ fluid $\% 20 \mathrm{in} \% 20$ COVID-19\%20patients.pdf.

7. Zheng MH, Boni L, Fingerhut A. Minimally invasive surgery and the novel coronavirus outbreak: lessons learned in China and Italy. Ann Surg. 2020 Mar 26. https://doi.org/10.1097/ SLA.0000000000003924. [Epub ahead of print].

8. Morris SN, Fader AN, Milad MP, et al. Understanding the "scope" of the problem: Why laparoscopy is considered safe during the COVID-19 pandemic. J Minim Invasive Gynecol. 2020 May-Jun;27(4):789-791. https://doi.org/10.1016/j.jmig. 2020.04.002. [Epub 2020 Apr 3].

9. SAGES Position Statement on endoscopy and PPE requirements during COVID-19 pandemic [Internet]. South African Gastroenterology Society. 2020. Available from: https://www. sages.co.za/newsletter_SAGES/PPE_Requirements.pdf. (Accessed 9 May 2020).

10.Resources for smoke and gas evacuation during open, laparoscopic, and endoscopic procedures [Internet]. SAGES. 2020. Available from: https://www.ges.org/resources-smokegas-evacuation-during-open-laparoscopic-endoscopicprocedures (Accessed 9 May 2020).

11. Mintz Y, Arezzo A, Boni L, et al. A low cost, safe and effective method for smoke evacuation in laparoscopic surgery for suspected coronavirus patients. Ann Surg. 2020 Apr 13. https:// doi.org/10.1097/SLA.0000000000003965. [Epub ahead of print]. 